\title{
Pharmacokinetics of Morphine in Rats with Adjuvant-induced Arthritis
}

\author{
YOSHIAKI KIMURA ${ }^{1,2}$, MIKA SHIBATA $^{1}$, MIKA TAMADA ${ }^{1}$, NORIYUKI OZAKI $^{3}$ and KUNIZO ARAI $^{1}$ \\ ${ }^{1}$ Faculty of Pharmacy, Institute of Medical, Pharmaceutical, and Health Sciences, and \\ ${ }^{3}$ Department of Functional Anatomy, Graduate School of Medical Sciences, \\ Kanazawa University, Kanazawa, Japan, \\ ${ }^{2}$ Suisen Pharmacy, Fukui Pharmaceutical Association, Eiheiji, Japan
}

\begin{abstract}
We investigated the in vivo dynamics and analgesic effect of morphine using an adjuvant-induced arthritis (AA) rat as a model of chronic inflammation. Morphine generally binds to $\mu$-opioid receptors in the brain to exert its effects. After several minutes, it is metabolized by glucuronidation via a UDP-glucuronosyltransferase (UGT). Here, we showed that in AA rats, UGT activity in liver microsomes was reduced. Morphine-free serum fractions in $A A$ rats were also decreased (control, 84.9\%; AA, 63.9\%) and the expression of ATP-binding cassette, sub-family $B$ $(M D R / T A P)$, member 1 (ABCB1), which plays a crucial role in morphine bile excretion, decreased to $23.0 \%$ that of the control group. However, we observed no significant difference between the $A A$ and control groups regarding blood concentrations of morphine and morphine-3glucuronide. In contrast, the analgesic effect of morphine increased 4-fold in AA rats. Our results showed that the pharmacokinetics of morphine is not changed, but the pharmacodynamics of morphine is enhanced in chronic inflammation
\end{abstract}

Morphine is commonly used to treat cancer-related pain and is also administered to control pain associated with inflammation. Inflammation and infection can modify the metabolism of numerous small molecules in both humans and rodents by altering the expression of proteins involved in the absorption, distribution, metabolism, and excretion of

This article is freely accessible online.

Correspondence to: Kunizo Arai, Faculty of Pharmacy, Institute of Medical, Pharmaceutical, and Health Sciences, Kanazawa University, Kakuma-machi, Kanazawa 920-1192, Japan. E-mail: arai@p.kanazawa-u.ac.jp

Key Words: Morphine, inflammation, adjuvant-induced arthritis, UDP-glucuronosyltransferase, ABCB1. numerous drugs, resulting in significantly increased or decreased potency and toxicity.

Morphine metabolism is carried out primarily by UDPglucuronosyltransferase (UGT), an enzyme expressed in the liver, small intestine, brain, and other organs. In the rat, morphine is glucuronidated by UGT2B1, a UGT isozyme which has a specific affinity for morphine-3-glucuronide (M3G) (1-4). Once glucuronidated, the morphine conjugate is then transported to and excreted by the kidney (in urine) and liver (in bile). Morphine and M3G are substrates of ATPbinding cassette transporters belonging to the family multidrug resistance-associated proteins. Morphine is transported by P-glycoprotein, encoded by the $A B C B 1$ gene, out of the brain capillary endothelium (5). M3G is transported across sinusoidal and canalicular membranes by several members of the ABC family, while morphine conjugates are transported by ATP-binding cassette transporters (ABC) C2 across the canalicular membrane into the bile and by ABCC3 across the sinusoidal membrane into the blood (6).

In animal models, infection and inflammation are associated with immediate and often dramatic alterations in the expression of liver-derived proteins. These inflammationassociated changes, known as acute-phase responses, can augment the plasma concentration of various drugs (e.g. propranolol, verapamil, or vancomycin), with the potential for toxic side-effects.

The present study was, therefore, designed to examine the pharmacokinetics of morphine and $\mathrm{M} 3 \mathrm{G}$ in a rat model of arthritis (AA) induced using complete Freund's adjuvant (CFA).

\section{Materials and Methods}

Chemicals and reagents. Morphine hydrochloride was purchased from Takeda Chemical Industries (Osaka, Japan). M3G was a generous gift from Professor Hideyuki Yamada, Kyushu University (Fukuoka, Japan). Diclofenac, naloxone, uridine 5'diphosphoglucronic acid (UDPGA), and alamethicin were purchased from Sigma-Aldrich (St. Louis, MO, USA). 
Animals. Male Lewis rats (7 to 10 weeks old; $170-200 \mathrm{~g}$ ) were purchased from Sankyo Laboratory Animal Co., Ltd. (Hamamatsu, Japan). Rats were housed under a 12-h light/dark cycle with free access to food and water. All animal procedures were carried out in accordance with the standards set forth in the Guidelines for the Care and Use of Laboratory Animals at the Takara-machi campus of Kanazawa University, and the experimental protocol was approved by the Institutional Animal Care and Use Committee of Kanazawa University, Japan (approval number: AP-153355).

Preparation of AA rats. Adjuvant was prepared from $100 \mathrm{mg}$ heatkilled Mycobacterium butyricum (Difco Laboratories, Detroit, MI, USA) suspended in $10 \mathrm{ml}$ of incomplete Freund's adjuvant (SigmaAldrich). A volume of $0.6 \mathrm{ml}$ of CFA mixture or $0.1 \mathrm{ml}$ of vehicle (mineral oil) was injected into the base of the tail of fifteen male Lewis rats under ketamine anesthesia. To evaluate the level of inflammation, hind paw thickness and the behavioral pain assays (by von Frey test) were measured. Animals were studied at 14 days (chronic phase) after injection of the adjuvant. For chronic-phase arthritis, severe inflammation was observed in systemic sites with associated hyperalgesia, increase of hind paw swelling, and weight loss. Arthritis was not induced in normal control rats and they received only the vehicle.

Microsome preparation. Male Lewis rat liver microsomes were prepared as previously described (7). Briefly, tissues were minced and rinsed in ice-cold homogenate buffer $(0.1 \mathrm{M}$ Tris- $\mathrm{HCl}, 0.1 \mathrm{M} \mathrm{KCl}, 1$ $\mathrm{mM}$ EDTA, $\mathrm{pH}$ 7.4) and homogenized in three volumes of $100 \mathrm{mM}$ phosphate buffer ( $\mathrm{pH}$ 7.4) containing $0.25 \mathrm{M}$ sucrose. The homogenate was centrifuged for $15 \mathrm{~min}$ at $9,000 \times g$. The supernatant was further centrifuged for $60 \mathrm{~min}$ at $105,000 \times \mathrm{g}$. The resulting microsomal pellet was resuspended in 1 volume of TGE buffer (10 mM Tris-HCl, $20 \%$ glycerol, $1 \mathrm{mM}$ EDTA, $\mathrm{pH}$ 7.4). Protein concentrations were determined using a Bio-Rad protein assay kit (Bio-Rad, Hercules, CA, USA) to adapt the assay from the standard-concentration range to a low-concentration $(<25 \mu \mathrm{g} / \mathrm{ml} ; 1-20 \mu \mathrm{g}$ total $)$.

Western blot analysis. Liver microsomal membrane proteins were extracted using the Mem-PER eukaryotic membrane protein extraction reagent kit (Thermo Fisher Scientific, Waltham, MA, USA) described as Kawase et al. (8). The membrane proteins were mixed with sample buffer, heated at $100^{\circ} \mathrm{C}$ for $5 \mathrm{~min}$, and electrophoresed on a $10 \%$ polyacrylamide gel. Proteins were then transferred to a polyvinylidene fluoride membrane (Immobilon-P; Millipore Corporation, Billerica, MA, USA). Immunoblotting was carried out using the following primary antibodies: rabbit antiABCB1 (E1Y7S) (Cell Signaling, Danvers, MA, USA) and mouse anti- $\beta$-actin (clone B11V08) (Acris Antibodies, San Diego, CA, USA). Detection of immune complexes was achieved using horseradish peroxidase-conjugated goat anti-rabbit or anti-mouse secondary antibodies (KPL, Milford, MA, USA) and ECL Prime Western blotting detection reagent (GE Healthcare, Piscataway, NJ, USA). Relative chemiluminescence was quantified using Image Quant LAS 4000 (GE Healthcare Bioscience, Tokyo, Japan).

Morphine glucuronosyltransferase activity. Measurement of morphine glucuronosyltransferase activity was performed as described previously by Hara et al. (9). Briefly, UDPGA was added to $0.2 \mathrm{ml}$ of reaction buffer [ $50 \mathrm{mM}$ Tris- $\mathrm{HCl}(\mathrm{pH} 7.4), 5 \mathrm{mM}$ $\mathrm{MgCl}_{2}, 5 \mathrm{mM}$ UDPGA, $25 \mu \mathrm{g} / \mathrm{ml}$ alamethicin, $0.25 \mathrm{mg} / \mathrm{ml}$ microsomal protein, and 25-200 $\mu \mathrm{M}$ morphine]. Termination was achieved by addition of $0.1 \mathrm{ml}$ ice-cold perchloric acid and incubated at $37^{\circ} \mathrm{C}$ for $30 \mathrm{~min}$. After removal of proteins by centrifugation at $10,000 \times g$ for $5 \mathrm{~min}$, components of the supernatant were separated by high performance liquid chromatography. Limits of detection of morphine and morphine 3- glucuronides were $5 \mathrm{fmol}$ and $20 \mathrm{fmol}$, respectively. The Dixon plots were used to determine the type of inhibition. Kinetic parameters were determined by nonlinear regression analysis using SigmaPlot 13 (Hulinks, Tokyo, Japan). All experiments were performed in triplicate.

Analgesic effects of morphine. The analgesic effects of morphine were evaluated using the Von Frey test, as described previously by Shinoda et al. (10). Briefly, the mechanical sensitivity of the plantar surface of the hind paw was assessed using Von Frey hairs. The von Frey hairs were pressed against the plantar surface of the hind paw and withdrawal response frequency was measured from five trials. Paw-withdrawal threshold was defined as the minimum pressure needed to evoke a response in at least $60 \%$ of the trials.

Tail flick test. tail flick tests were used to determine the loss of antinociceptive effectiveness of morphine. Animals were placed separately on an electrically-heated surface $\left(55 \pm 1^{\circ} \mathrm{C}\right)$. tail flick latency was measured by recording the time from the onset of heat stimulation to withdrawal of the tail from the heat source. To avoid injury to the tail, a cut-off time of $30 \mathrm{~s}$ was employed. The analgesic effect was expressed as a percentage of the maximum possible effect (MPE): \% MPE=(post-drug threshold - pre-drug threshold)/ (maximum threshold - pre-drug threshold) $\times 100$, where pre-drug threshold was the mean of the tail-withdrawal threshold in the salinetreated (vehicle) group, post-drug threshold was the tail-withdrawal threshold of each animal treated with drug, and maximum threshold was the cut-off value. The area under each effect-versus-time curve (AUC) was determined by trapezoidal integration and these were compared using Wilcoxon matched-pairs test.

Plasma protein binding. Plasma filtration was used to determine bound and unbound fractions of morphine. After incubation of the rat serum injectate solution at $37^{\circ} \mathrm{C}$ for $5 \mathrm{~min}, 0.8 \mathrm{ml}$ of the sample was transferred immediately to a Centrifree tube (Amicon Co., Danvers, MA, USA). The tube was then centrifuged at $1,500 \times g$ for $15 \mathrm{~min}$ at $37^{\circ} \mathrm{C}$. The unbound fraction of morphine was estimated directly from the ratio of drug concentration in filtrate to the total drug concentration in the original injectate solution. The percentage of plasma protein binding was calculated based on the concentration of test compounds in the plasma and buffer chambers.

Data analysis and statistics. A $t$-test or a one-way analysis of variance (ANOVA) was used to compare two or many groups, respectively (SPSS statistics 23; IBM Corp., Armonk, NY, USA). Some groups were more numerous because control groups were repeated and the data were pooled when there was no significant differences. Inter-group differences were analyzed by ANOVA. In all cases, the accepted significance level was $p<0.05$.

\section{Results}

Validation of AA rats and evaluation of systemic inflammation. We measured hyperalgesia (by von Frey test) (Figure 1A), hind paw swelling (Figure 1B) and body 


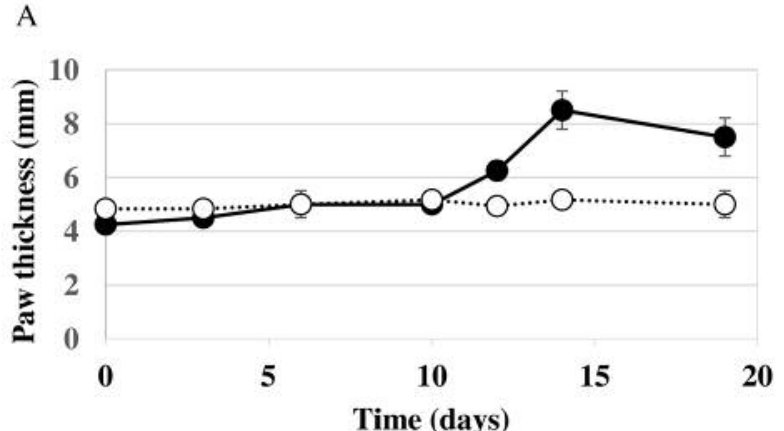

B
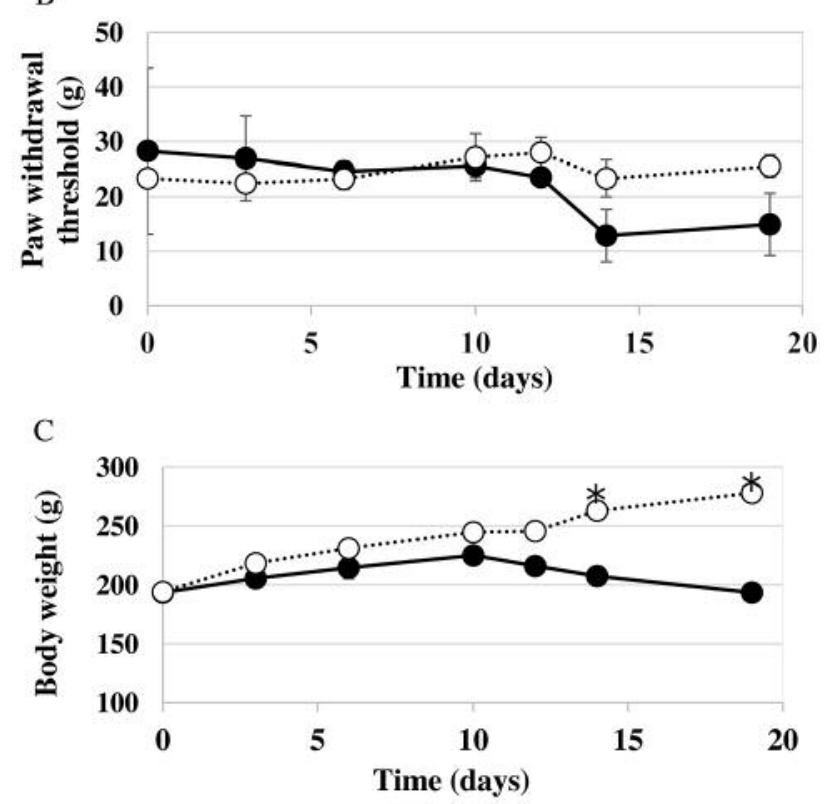

Figure 1. Adjuvant-induced arthritis (AA) generates paw edema (A), mechanical hyperalgesia $(B)$, and weight loss $(C)$. Data represent the mean $\pm S D,(n=3) . \triangle A A, \bigcirc$ Control. $*$ Statistically significant difference compared with control rats at $p<0.05$.

weight (Figure 1C) in AA rats or rats treated with vehicle (control). In AA rats, we observed an increase in hyperalgesia, while swelling was seen on day 14 of adjuvant administration. The weight of AA rats was significantly reduced compared to that of the control group as of day 12. Symptoms common to systemic inflammation such as hyperalgesia, increase of hind paw swelling, and weight loss were confirmed in the CFA-administered group, which is consistent with previous reports $(11,12)$. These results suggest that our rat model is valid with respect to induction of AA pathogenesis.

Effect of CFA-induced inflammation on UGT activity, ABCBI protein expression, and serum protein binding of morphine.

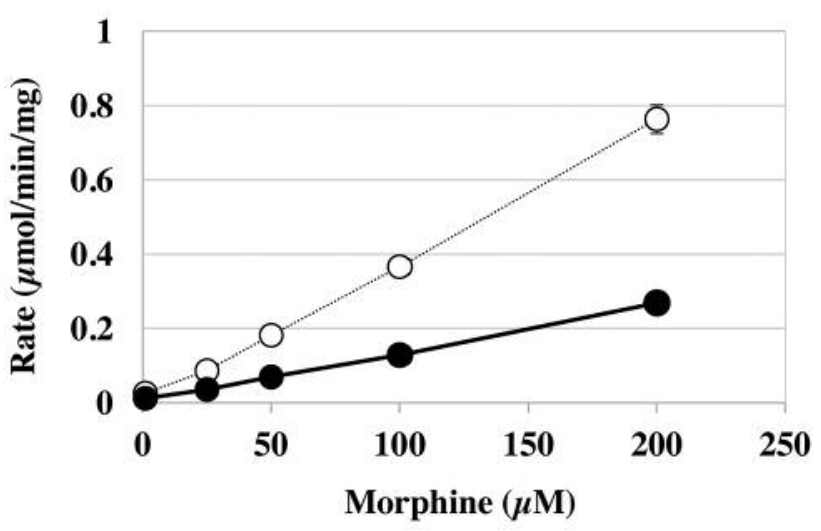

Figure 2. Representative plot of velocity versus morphine concentration for the 3-glucuronidation of morphine in rat liver microsomes. The solid lines are computer-generated curves for best fit. Data represent the mean $\pm S D$. Adjuvant-induced arthritis $(A A), \bigcirc$ Control.

Table I. Michaelis-Menten parameters for 3-glucuronidation of morphine by rat liver microsomes. Date are shown as mean.

\begin{tabular}{lcc}
\hline Group & Vmax $(\mathrm{mmol} / \mathrm{min} / \mathrm{mg})$ & $\mathrm{Km}(\mu \mathrm{mol} / \mathrm{l})$ \\
\hline Control & 286.7 & 75.93 \\
Adjuvant-induced arthritis & 26.62 & 19.94 \\
\hline
\end{tabular}

Using morphine as a substrate, we determined the kinetics of UGT activity in control and AA rats in liver microsomes. In the AA rats, UGT activity, as well as its Vmax and $\mathrm{Km}$ (Figure 2, Table I), decreased compared with those of the control group.

Liver ABCB1 levels were significantly lower in AA rats than in the control rats (Figure 3). The morphine-free fraction was $84.9 \%$ in the control group and $63.9 \%$ in the AA rat model, and, in AA rats, and was significantly lower compared with that of the control group (Figure 4).

Morphine and M3G pharmacokinetics. We found that the plasma concentration of morphine was lower in AA rats than in the control group. However, the difference was not statistically significant. Furthermore, the AUC, mean residence time, total body clearance, and volume of distribution of morphine were similar between AA and control rats. Yet the elimination rate constant was higher in AA rats, but the difference again was not statistically significant. We found no difference between formations of M3G in either the AA or control groups (Figure 5). 


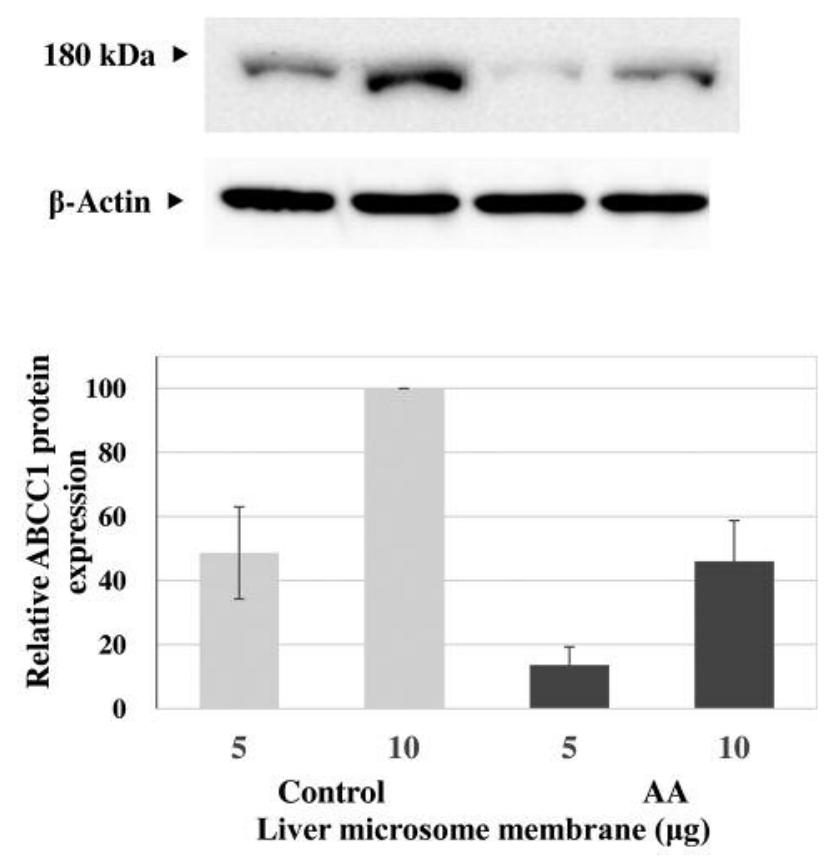

Figure 3. Changes in relative protein levels of ATP-binding cassette transporters $(A B C) C 1$ in microsomal proteins from livers of rats with adjuvant-induced arthritis (AA). Densitometric quantification of $A B C C 1$ was performed and expression values were normalized to those of $\beta$-actin. Representative blots of two independent experiments are shown $(n=3)$.

Anti-nociceptive effect of morphine using tail flick test. In AA rats, the analgesic effect of morphine was maximal 30$60 \mathrm{~min}$ after administration and $30 \mathrm{~min}$ after administration in the control group. Morphine increased the MPE, and the peak of MPE at $60 \mathrm{~min}$. The AUC of MPE in AA rats was found to be four times higher than that of the control animals (Figure 6).

\section{Discussion}

Recently, chronic inflammation has been increasingly identified as a pathological condition common to various chronic diseases. The molecular mechanisms and pathophysiological significance of chronic inflammation have been intensively studied in arteriosclerosis, autoimmune diseases, obesity, cancer, and neurodegenerative diseases (13). For example, in atherosclerosis, it has been observed that inflammation commences via complex cell-cell interactions of inflammatory cells such as macrophages in the blood vessel wall (13). Therefore, this disease has been now widely characterized as a chronic inflammatory illness. Furthermore, serum levels of inflammatory cytokines are elevated in patients with severe heart failure, which

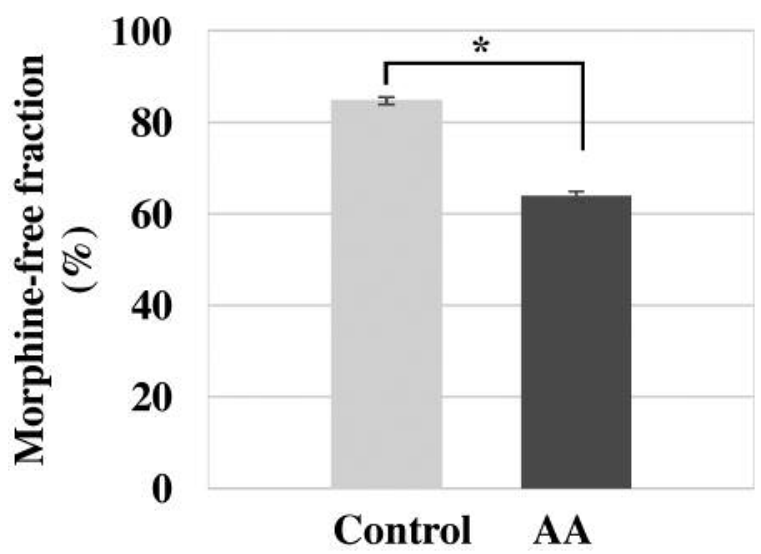

Figure 4. Binding of morphine to serum protein in rats with adjuvantinduced arthritis $(A A)$ and controls. ${ }^{*}$ Statistically significant difference compared to controls at $p<0.05$.

correlates with its clinical severity. It has also been observed that prolongation of inflammation in atrial tissues is involved in the development of atrial fibrillation. Finally, inflammation has also been shown to play an important role in chronic kidney disease (CKD), as well as in type 2 diabetes and metabolic syndrome (13).

Previous studies have detailed the effects of inflammatory cytokines on drug-metabolizing enzyme activity and drugtransporter function. Cytochrome P-450 (CYP) expression levels have been shown to decrease after secretion of inflammatory cytokines such as Interleukin (IL)-1, IL-6, tumor necrosis factor $\alpha$, and interferon in animal models of lipopolysaccharide (LPS)-induced inflammation (14). It has been proposed that the regulation of CYP expression levels by cytokines occurs transcriptionally. For example, pregnane $\mathrm{X}$ receptor (PXR) and CYP3A expression levels were found to be decreased in the livers of mice treated with LPS (15). Therefore, regulation of PXR expression by inflammatory cytokines might result in a decrease in CYP activity. However, multiple mechanisms might exist whereby inflammatory cytokines regulate gene expression, as CYP activity is decreased due to inflammation (16). Additionally, several groups have reported on metabolic enzymes other than CYP. When LPS is administered to rats, the expression levels and activity of a flavin-containing monooxygenase decline, and this effect is restored by inhibiting nitric oxide (NO) synthase. Therefore, NO may be involved in regulating flavin-containing monooxygenase activity during inflammation (17). A reduction in the expression levels of UGT has also been reported, while inflammatory cytokines have been shown to reduce drugtransporter function in both animal and human cellular models (18). Similar to its effect on the expression of metabolic 


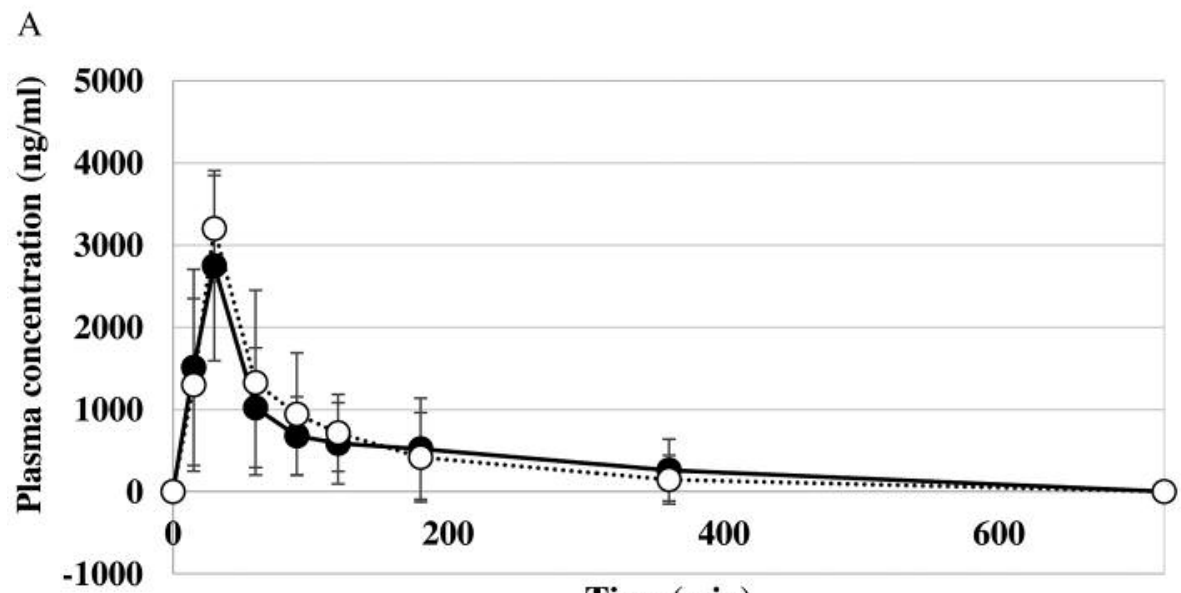

Time (min)

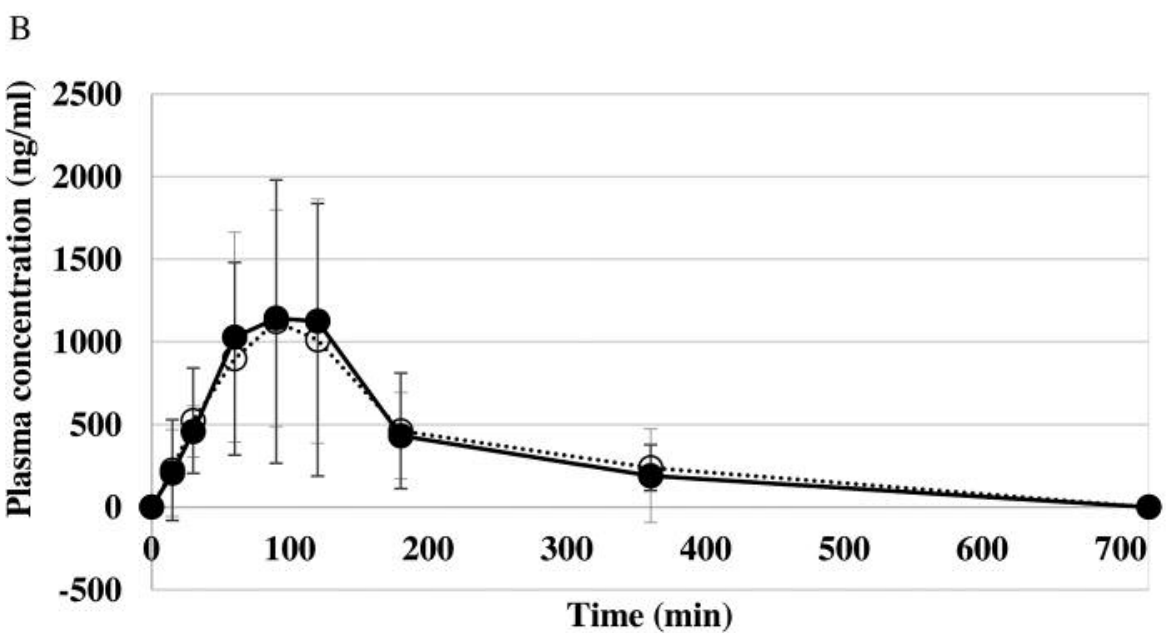

Figure 5. Plasma concentration time course of morphine (A) and morphine-3-glucuronide $(B)$ in rats with adjuvant-induced arthritis (AA) after a single administration of morphine $(5 \mathrm{mg} / \mathrm{kg})$. Data represent the mean $\pm S D(n=3)$.*Statistically significant differences compared to control groups at $p<0.05$.

Table II. Pharmacokinetics parameters of morphine and morphine-3-glucuronide after a single administration of morphine (5 mg/kg) in adjuvantinduced arthritis rats.

\begin{tabular}{|c|c|c|c|c|c|c|c|}
\hline Compound & Group & $\mathrm{AUC}, \mathrm{mg} / \mathrm{ml} \mathrm{min}$ & MRT, min & VRT, min min & $t_{1 / 2}, \min$ & CLtot, $1 / \mathrm{h} / \mathrm{kg}$ & Vdss, $1 / \mathrm{kg}$ \\
\hline \multirow[t]{2}{*}{ Morphine } & Control & 197.7 & 135.3 & 6,953 & 102.1 & 0.02238 & 3.986 \\
\hline & Adjuvant-induced arthritis & 247.9 & 116.0 & 9,911 & 199.8 & 0.01542 & 3.721 \\
\hline \multirow[t]{2}{*}{ Morphine-3-glucuronide } & Control & 197.7 & 135.4 & 6,953 & 102.1 & & \\
\hline & Adjuvant-induced arthritis & 197.9 & 142.3 & 8,044 & 126.2 & & \\
\hline
\end{tabular}

AUC: Area under the blood concentration time curve; MRT: mean residence time; VRT: variance of residence time; t1/2: hafe-life; CLtot: total body clearance; Vdss: steady state volume of distribution.

enzymes, the expression levels of solute carrier transporters such as the organic anion transporter and the organic cation transporter, are reduced by inflammatory cytokines (8).
In this study, we showed that liver ABCB1 levels were significantly lower in AA rats. Kawase et al. demonstrated that the membrane localization of $\mathrm{ABC}$ transporters in liver 
decreased without change of total expression levels in AA rats and the interactions between radixin, which is involved in localization of some $\mathrm{ABC}$ transporters in canalicular membrane, and $\mathrm{ABC}$ transporters were decreased in inflammation in AA (19).

Modulation of the expression of metabolic enzymes and transporters, and changes in the pharmacokinetics of morphine metabolism have also been reported in several models of disease. In rats treated with streptozotocin, known to induce both diabetes and bile duct ligation-induced cholestasis (20), expression levels of UGT2B1, ABCC2, and ABCC3 were found to be altered and biliary excretion of $\mathrm{M} 3 \mathrm{G}$ was reduced. Additionally, plasma morphine concentrations were found to be lower in mice treated with streptozotocin compared with those of the control group. This effect was due to an increase in the distribution of morphine since albumin levels were comparable between the two groups. In this study, elimination of morphine and the elimination half-life were also similar. These results suggest that morphine levels may be lower in diabetic compared with non-diabetic patients and may explain, in part, why morphine is less potent in diabetic patients.

Non-alcoholic steatohepatitis (NASH) is the most severe stage of progressive nonalcoholic fatty liver disease (NAFLD) (21). ABCC2 shares substrate specificity with $\mathrm{ABCC} 3$, which is a sinusoidal efflux transporter whose expression is increased in NASH, while the expression of a subset of UGT isoforms appears to be differentially regulated during NAFLD (21).

Differences in the pharmacokinetics of several drugs in patients with rheumatoid arthritis and Crohn's disease have been reported. During inflammation, the plasma level of $\alpha 1$ acid glycoprotein, a protein known to bind a variety of drugs, increases, causing the release and clearance rate of these drugs to decrease, leading to an increase in blood concentration and in AUC. In agreement with this, patients with rheumatoid arthritis and Crohn's disease have shown decreases in free fractions of blood when treated with either propranolol, verapamil, or vancomycin $(22,23)$.

In our AA rat model, we found that UGT activity decreased $25 \%$ compared with that of the control rats. Furthermore, we observed decreases in ABCB1 expression level in the liver. In addition, the morphine free serum fraction was $84.9 \%$ in the control group and only $63.9 \%$ in AA rats. Previous studies have suggested that $\alpha 1$-acid glycoprotein production increases due to inflammation (24) and that the protein binding rate of morphine, a basic drug, increases. However, in our study, inflammation reduced the activity of UGT and expression of $\mathrm{ABCB} 1$, and negatively affected the pharmacokinetics of morphine and its metabolite, M3G.

Although the pharmacokinetics of morphine did not change in AA rats, the analgesic effect of morphine was enhanced. It has been reported that in AA rats expression of $\mu$-opioid receptors in the central nervous system and the analgesic effect of morphine increased (25). These data corroborate our

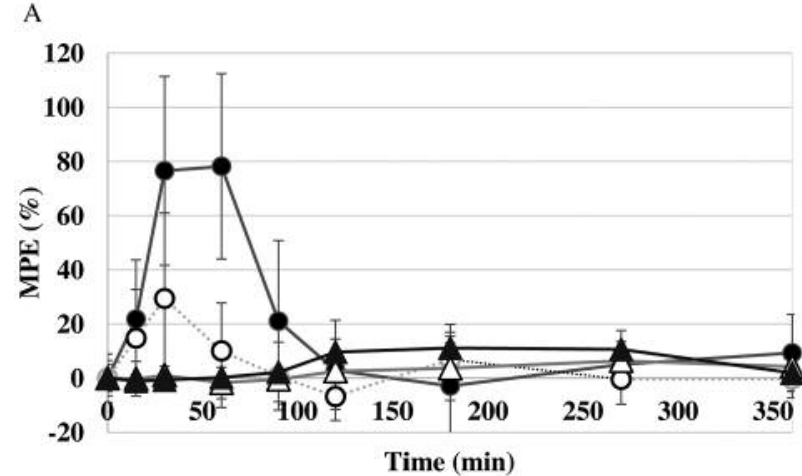

B

$*$

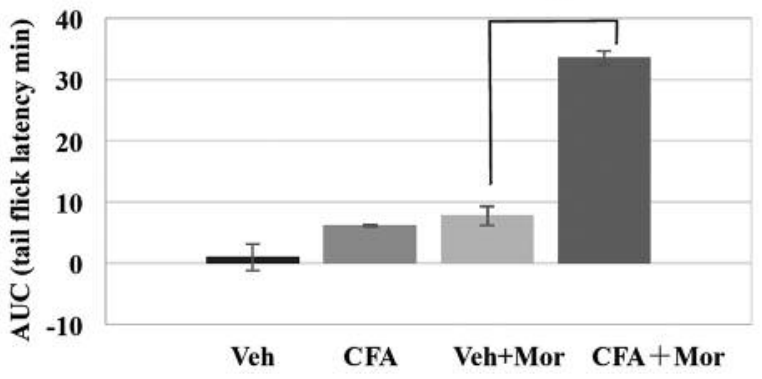

Figure 6. Anti-nociceptive effects of morphine (Mor) in rats with arthritis induced by complete Freund's adjuvant (CFA) and vehicletreated controls (Veh) are presented as a percentage of the maximum possible effect (MPE). CFA+Mor, $\bigcirc$ Veh+Mor, $\triangle$ CFA, $\triangle \operatorname{Veh}(A)$ and The area under each effect-versus-time curve (AUC) for the tail flick test (B). AUC calculations were performed using the trapezoidal method. Data represent the mean $\pm S D(n=5)$. *Statistically significant difference compared with control groups at $p<0.05$.

observation that in the presence of systemic inflammation induced by administration of an adjuvant, sensitivity to morphine was enhanced, likely by increased expression of $\mu$ opioid receptors in the central nervous system.

We previously showed that glucuronidation of morphine is not inhibited even when diclofenac, which strongly inhibits UGT activity in vitro, is administered in combination with morphine in vivo (7). In that study, glucuronidation of morphine was similar between mice treated with either diclofenac or vehicle, despite a decrease in UGT activity due to inflammation. These results suggest that UGT activity had no effect on morphine metabolism. Opioid-related adverse events occur in approximately $15 \%$ of patients postoperatively (26). Morphine-related adverse reactions are generally associated with genetic polymorphisms in genes encoding factors related to morphine pharmacokinetics such as glucuronidation in the liver, elimination (efflux from blood-brain barrier), and pharmacodynamics ( $\mu$-opioid 
receptor) (27). Our results suggest that side-effects related to elevated blood concentrations of morphine cannot be caused by inhibition of morphine-specific UGT activity.

\section{Conflicts of Interest}

The Authors have no conflict of interest directly relevant to the content of this article.

\section{References}

1 Kuo CK, Hanioka N, Hoshikawa Y, Oguri K and Yoshimura $\mathrm{H}$ Species difference of site-selective glucuronidation of morphine. J Pharmacobiodyn 14: 187-193, 1991.

2 Salem A and Hope W: Role of morphine glucuronide metabolites in morphine dependence in the rat. Pharmacol Biochem Behav 57: 801-807, 1997.

3 Hasegawa Y, Kishimoto S, Takahashi H, Inotsume N, Takeuchi $\mathrm{Y}$ and Fukushima S: Altered expression of MRP2, MRP3 and UGT2B1 in the liver affects the disposition of morphine and its glucuronide conjugate in a rat model of cholestasis. J Pharm Pharmacol 61: 1205-1210, 2009.

4 Dzierlenga AL, Clarke JD, Hargraves TL, Ainslie GR, Vanderah TW, Paine MF and Cherrington NJ: Mechanistic basis of altered morphine disposition in nonalcoholic steatohepatitis. J Pharmacol Exp Ther 352: 462-470, 2015.

5 Zong J and Pollack GM: Morphine anti-nociception is enhanced in Mdr1a gene-deficient mice. Pharm Res 17: 749-753, 2000.

6 van de Wetering K, Zelcer N, Kuil A, Feddema W, Hillebrand M, Vlaming ML, Schinkel AH, Beijnen JH and Borst P: Multidrug resistance proteins 2 and 3 provide alternative routes for hepatic excretion of morphine-glucuronides. Mol Pharmacol 72: 387-394, 2007.

7 Kimura K, Muryoi K, Shibata M, Ozaki N and Arai K: In vivo interaction of morphine and diclofenac. Pharmacology \& Pharmacy 7: 493-503, 2016.

8 Kawase A, Norikane S, Okada A, Adachi M, Kato Y and Iwaki M: Distinct alterations in ATP-binding cassette transporter expression in liver, kidney, small intestine, and brain in adjuvantinduced arthritic rats. J Pharm Sci 103: 2556-2564, 2014.

9 Hara Y, Nakajima M, Miyamoto K and Yokoi T: Morphine glucuronosyltransferase activity in human liver microsomes is inhibited by a variety of drugs that are co-administered with morphine. Drug Metab Pharmacokinet 22: 103-112, 2007.

10 Shinoda K, Hruby V and Porreca F: Antihyperalgesic effects of loperamide in a model of rat neuropathic pain are mediated by peripheral $\delta$-opioid receptors. Neurosci Lett 411: 143-146, 2007.

11 Stein C, Millan MJ and Herz A: Unilateral inflammation of the hindpaw in rats as a model of prolonged noxious stimulation: Alterations in behavior and nociceptive thresholds Pharmacology Biochemistry \& Behavior 31: 445-451, 1998.

12 Donaldson LF, Seckl JR and McQueen DS: A discrete adjuvantinduced monoarthritis in the rat: effects of adjuvant dose. Journal of Neuroscience Methods 46: 5-10, 1993.

13 Hotmisligil GS: Inflammation and metabolic disorders. Nature 444: 860-867, 2006.

14 Gu X, Ke S, Liu D, Sheng T, Thomas PE, Rabson AB, Gallo MA, Xie W and Tian Y: Role of NF-kappaB in regulation of PXR-mediated gene expression: a mechanism for the suppression of cytochrome P-450 3A4 by proinflammatory agents. J Biol Chem 281: 17882-7889, 2006.

15 Moriya N, Kataoka H, Fujino H, Nishikawa J and Kugawa F: Effect of lipopolysaccharide on the xenobiotic-induced expression and activity of hepatic cytochrome P450 in mice. Biol Pharm Bull 35: 473-480, 2012.

16 Teng S and Piquette-Miller M: The involvement of the pregnane $X$ receptor in hepatic gene regulation during inflammation in mice. J Pharmacol Exp Ther 312: 841-848, 2005.

17 Zhang J, Chaluvadi MR, Reddy R, Motika MS, Richardson TA, Cashman JR and Morgan ET: Hepatic flavin-containing monooxygenase gene regulation in different mouse inflammation models. Drug Metab Dispos 37: 462-468, 2009.

18 Richardson TA, Sherman M, Kalman D and Morgan ET: Expression of UDP-glucuronosyltransferase isoform mRNAs during inflammation and infection in mouse liver and kidney. Drug Metab Dispos 34: 351-353, 2006.

19 Kawase A, Sakata M, Yada N, Nakasaka M, Shimizu T, Kato Y and Iwaki M: Decreased radixin function for ATP-binding cassette transporters in liver in adjuvant-induced arthritis rats. J Pharm Sci 103: 4058-4065, 2014.

20 Hasegawa Y, Kishimoto S, Takahashi H, Inotsume N, Takeuchi $\mathrm{Y}$ and Fukushima S: Altered expression of MRP2, MRP3 and UGT2B1 in the liver affects the disposition of morphine and its glucuronide conjugate in a rat model of cholestasis. J Pharm Pharmacol 61: 1205-1210, 2009.

21 Marra F, Gastaldelli A, Svegliati Baroni G, Tell G and Tiribelli C: Molecular basis and mechanisms of progression of nonalcoholic steatohepatitis. Trends Mol Med 14: 72-81, 2008.

22 Mayo PR, Skeith K, Russell AS and Jamali F: Decreased dromotropic response to verapamil despite pronounced increased drug concentration in rheumatoid arthritis. Br J Clin Pharmacol 50: 605-613, 2000.

23 Ling S, Lewanczuk RZ, Russell AS, Ihejirika B and Jamali F: Influence of controlled rheumatoid arthritis on the action and disposition of verapamil: focus on infliximab. J Clin Pharmacol 49: 301-311, 2009.

24 Sanaee F, Clements JD, Waugh AW, Fedorak RN, Lewanczuk R and Jamali F: Drug-disease interaction: Crohn's disease elevates verapamil plasma concentrations but reduces response to the drug proportional to disease activity. Br J Clin Pharmacol 72: 787-797, 2011.

25 Zaringhalam J, Manaheji H, Mghsoodi N, Farokhi B and Mirzaiee $\mathrm{V}$ : Spinal mu-opioid receptor expression and hyperalgesia with dexamethasone in chronic adjuvant-induced arthritis in rats. Clin Exp Pharmacol Physiol 35: 1309-1315, 2008.

26 Kessler ER, Shah M, Gruschkus SK and Raju A: Cost and quality implications of opioid-based postsurgical pain control using administrative claims data from a large health system: opioid-related adverse events and their impact on clinical and economic outcomes. Pharmacotherapy 33: 383-391, 2013.

27 Fujita K, Ando Y, Yamamoto W, Miya T, Endo H, Sunakawa Y, Araki K, Kodama K, Nagashima F and Ichikawa W: Association of UGT2B7 and ABCB1 genotypes with morphine-induced adverse drug reactions in Japanese patients with cancer. Cancer Chemother Pharmacol 65: 251-258, 2010.

Received June 17, 2017

Revised July 11, 2017

Accepted July 12, 2017 